\title{
A Patient with Duodenal Mucinous Adenocarcinoma Presenting as a Laterally Spreading Tumor
}

\author{
Myung Joon Chae, II Hyun Baek, Yu Mi Oh, Jun Uk Lim, Jung Won Jeon, Hyun Phil Shin, Kwang Ro Joo and Joung II Lee \\ Department of Gastroenterology, Kyung Hee University Hospital at Gangdong, Kyung Hee University School of Medicine, Seoul, Korea
}

Primary duodenal carcinoma is rare. Duodenal mucinous adenocarcinoma (DMA) is even rarer, and its associated manifestations and typical endoscopic or imaging findings are not well characterized. Herein, we report a case of primary DMA in an asymptomatic 58-year-old man who visited our hospital for a regular health screening. Upper endoscopy revealed an approximately 4-cm lesion in the second portion of the duodenum, but the mass was not visualized on computed tomography. Biopsies revealed a tubular adenoma that was subsequently resected. Frozen biopsies demonstrated DMA with a background of low-grade tubular adenoma for which we performed Roux-en-Y duodenojejunostomy and jejunojejunostomy. To our knowledge, this is the first report of a patient with DMA in Korea. Clin Endosc 2015;48:336-339

Key Words: Adenocarcinoma; Mucinous; Duodenal neoplasms; Adenoma

\section{INTRODUCTION}

Primary duodenal mucinous adenocarcinoma (DMA) is very rare. Although the small intestine comprises about $75 \%$ of the length and $>90 \%$ of the surface area of the gastrointestinal (GI) tract, small bowel malignancies account for only $3 \%$ of all GI tract neoplasms and approximately $0.5 \%$ of all cancers in the United States. ${ }^{1}$ Adenocarcinomas represent 25\% to $40 \%$ of small bowel cancers, and the incidence of adenocarcinoma is the highest in the duodenum. ${ }^{2}$ Mucinous adenocarcinoma, a type of cancer that arises from epithelial cells and produces copious amounts of mucin, has been described in many internal organs including the lungs, ovary, pancreas, appendix, stomach, colon, and rectum, but few cases of DMA have been reported. ${ }^{3,4}$

Received: August 19, 2014 Revised: October 14, 2014

Accepted: October 21, 2014

Correspondence: Il Hyun Baek

Department of Gastroenterology, Kyung Hee University Hospital at Gangdong, Kyung Hee University School of Medicine, 892 Dongnam-ro, Gangdong-gu, Seoul 134-727, Korea

Tel: +82-2-440-6152, Fax: +82-2-440-8150, E-mail: topcolon@nate.com

(c) This is an Open Access article distributed under the terms of the Creative Commons Attribution Non-Commercial License (http://creativecommons.org/ licenses/by-nc/3.0) which permits unrestricted non-commercial use, distribution, and reproduction in any medium, provided the original work is properly cited.
Herein, we describe a patient who underwent Roux-en-Y duodenojejunostomy and jejunojejunostomy for the treatment of DMA.

\section{CASE REPORT}

A 58-year-old man with no significant history of medical illness visited our hospital for a regular health screening. He underwent upper endoscopy, during which an approximately $4-\mathrm{cm}$ pinkish mass filling $>70 \%$ of the lumen of the second portion of the duodenum was noted. The mass was edematous and extended laterally along the side opposite the ampulla. The surface of the mass was irregular and nodular. The tumor was soft and it bled easily at the touch. No mucin or exudates were seen on the lesion (Fig. 1A). Multiple biopsies revealed a tubular adenoma. The patient was admitted to our hospital for further evaluation and surgery.

On admission, the patient's resting blood pressure was 130/80 mm Hg. He had no significant family medical history, and no symptoms such as abdominal pain, weight loss, or dyspepsia. The results of physical examination were unremarkable. Laboratory data were as follows: white blood cell count, $6,300 / \mathrm{mm}^{3}$ with $64.7 \%$ neutrophils; hemoglobin level, 

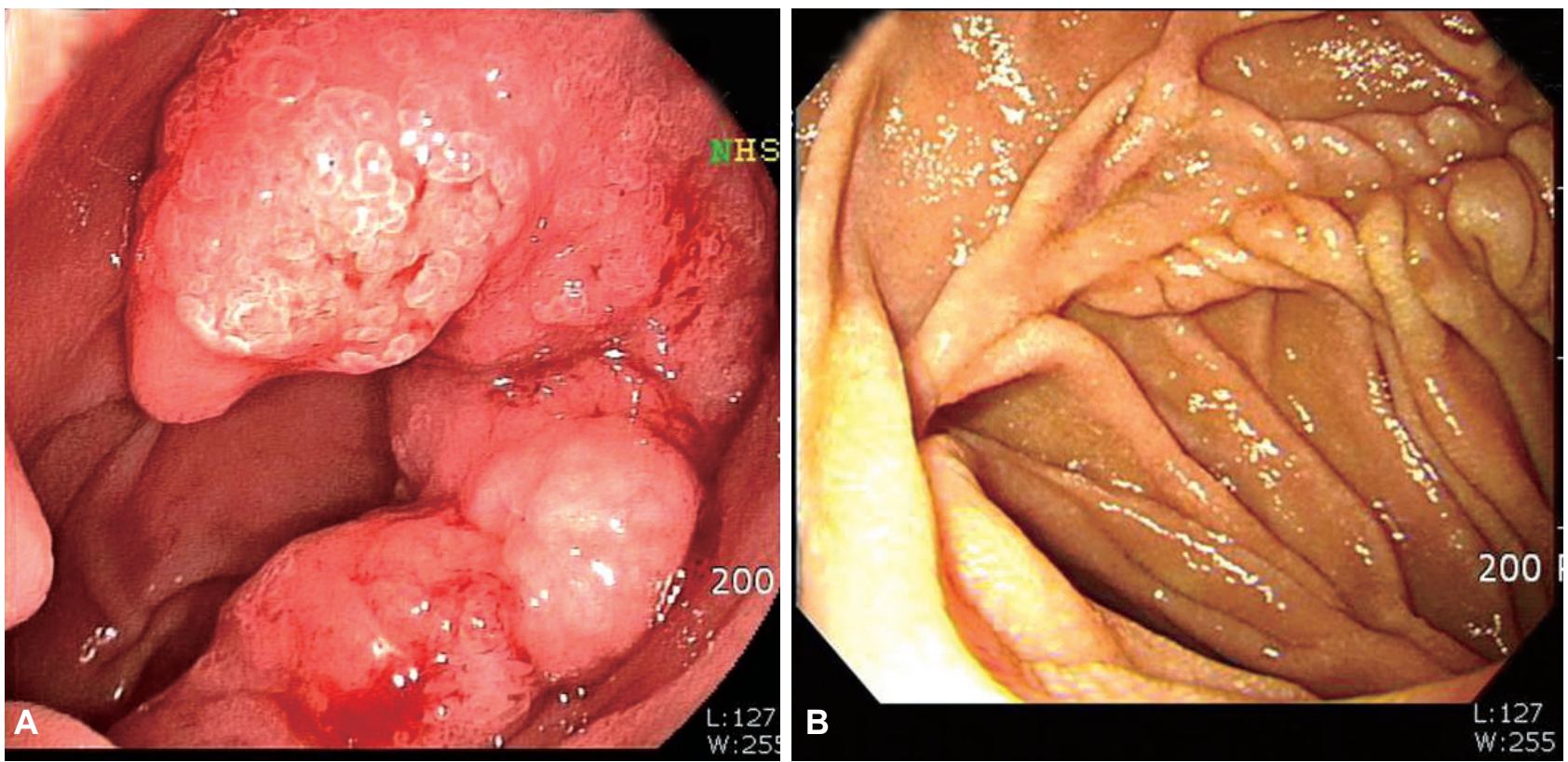

Fig. 1. Esophagogastroduodenoscopy. (A) A laterally spreading polyp with an irregular surface is visible in the second portion of the duodenum. (B) Follow-up endoscopy after 3 months, showing no evidence of recurrence or abnormal findings at the anastomosis within the duodenum.
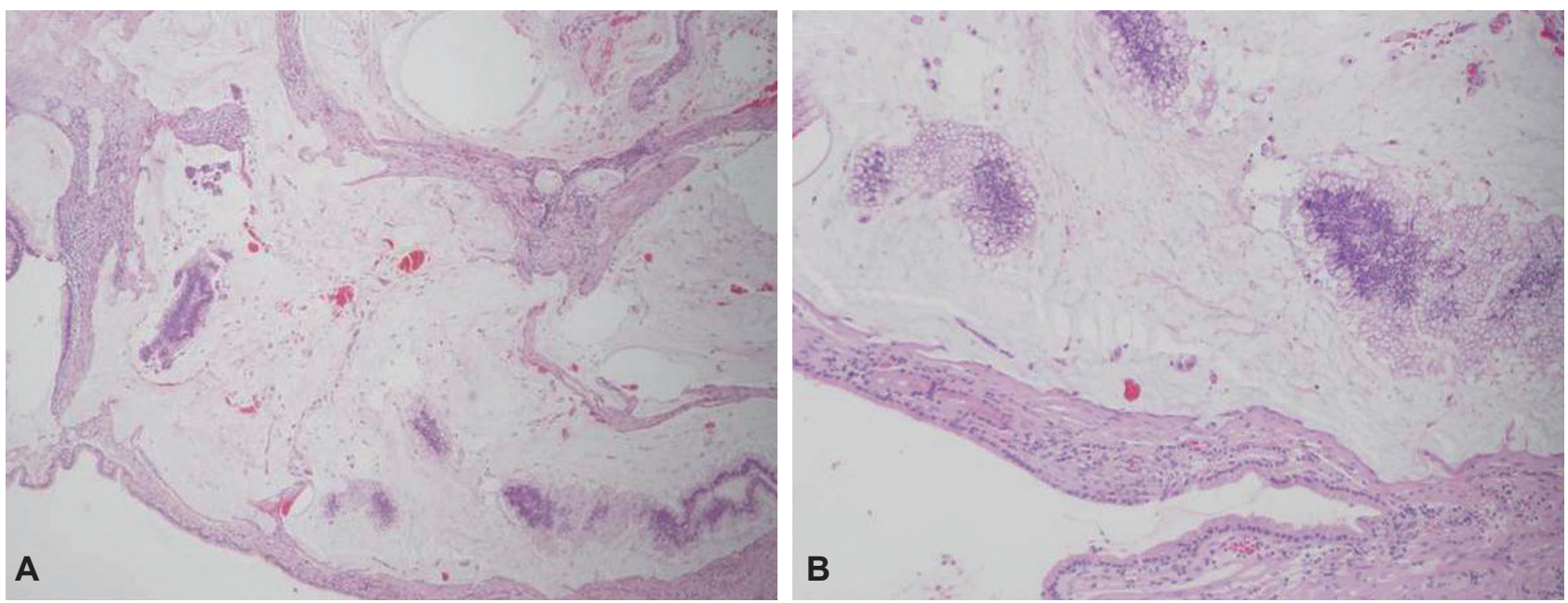

Fig. 2. Duodenal biopsy histology. These show malignant cells floating in large pools of mucin (A, H\&E stain, $\times 40$; $B, H \& E$ stain, $\times 100)$.

$15.6 \mathrm{~g} / \mathrm{dL}$; mean corpuscular volume, $96.6 \mathrm{fL}$; mean corpuscular hemoglobin concentration, $33.5 \mathrm{~g} / \mathrm{dL}$; and platelet count, $246,000 / \mathrm{mm}^{3}$. Electrolyte levels and renal and liver functions were all normal. Tests for tumor markers such as carcinoembryonic antigen and carbohydrate antigen revealed no abnormalities, and fecal occult blood test results were negative. Abdominal computed tomography failed to reveal the duodenal mass, and no enlargement of lymph nodes was seen. Findings associated with the liver, pancreas, gallbladder, spleen, kidneys, colon, and rectum were unremarkable, and chest radiography findings were normal.

Two days after his admission, the patient's adenoma was resected. A frozen biopsy showed a $0.7-\mathrm{cm}$ DMA and a background composed of low-grade tubular adenoma with multiple small high-grade foci and in situ carcinoma lesions (Fig. 2). The tumor had invaded the submucosa but not the lymphovascular system, and the resection margins were negative. We then performed Roux-en-Y duodenojejunostomy and jejunojejunostomy. Three months after the surgery, upper endoscopy findings were unremarkable at the duodenal anastomosis site (Fig. 1B). The patient was doing well without any evidence of recurrence at his 1-year follow-up visit. 


\section{DISCUSSION}

Primary small bowel adenocarcinoma (SBA) is a rare malignancy. Compared to colorectal cancer, little is known about the pathogenesis of SBA. SBA is associated with familial adenomatous polyposis, Lynch syndrome, Peutz-Jeghers syndrome, celiac sprue, and Crohn disease. ${ }^{5}$ The median age at diagnosis of SBA is during the sixth decade of life. ${ }^{6}$ In the USA, there are approximately 5,300 new cases and 1,100 deaths from SBA per year. In Europe, there are approximately 3,600 new cases of SBA every year, and the incidence rate is 5.7 cases per million. ${ }^{8}$ The incidence of SBA is increasing, mainly because of an associated increase in duodenal tumors.'

It can be difficult to detect SBA owing to its rarity and nonspecific symptoms. However, $43 \%$ to $66 \%$ of patients with SBA have abdominal pain at the time of diagnosis, and they are often diagnosed during emergencies involving occlusion or bleeding. The diagnosis of SBA is usually made at an advanced stage, and $35 \%$ of patients already have metastasis when diagnosed. ${ }^{10,11}$ Therefore, early diagnosis and resection are important for improved outcomes. Negative prognostic factors for SBA include poor tumor differentiation, positive surgical margins, lymph node metastases, extramural venous spread, tumor invasion depth, and Crohn disease. ${ }^{12}$

Mucinous adenocarcinoma is identified when mucus is present within $>50 \%$ of an adenocarcinoma, according to the World Health Organization. ${ }^{13}$ Mucinous adenocarcinoma may arise at various sites, and the prognostic significance of this subtype remains uncertain.

Yasuda et al. ${ }^{14}$ published a clinicopathological study of mucinous gastric carcinoma (MGC) in which most cases were detected during the advanced stage, and the biological behavior of advanced MGC did not differ from that of other gastric cancers. In their analysis, the majority of MGC cases were $\geq 5 \mathrm{~cm}$ (100\%), positive for lymph node metastasis ( $88 \%$ ), and positive for serosal invasion (76\%). Additionally, their study showed that the mucinous histological type was not an independent prognostic indicator, whereas the prognoses of mucinous carcinoma of the colon and rectum were unfavorable compared with other histological types. ${ }^{15}$ The prognosis, clinical features, and typical endoscopic findings of DMA remain unclear owing to the rarity of this disease.

Regarding early MGC, restriction to the mucosa is very rare. Hirota et al. ${ }^{16}$ reviewed the clinicopathological features of 6,288 cases of gastric carcinoma. Among these, MGC comprised 228 cases and early MGC comprised 15 cases, but the frequency of early mucosal MGC was only $0.4 \%(1 / 228)$. These findings may be related to the manner in which MGC develops, as it is thought to arise as a typical gastric carcino$\mathrm{ma}$ at an early stage and produce mucin later. ${ }^{17}$ The typical endoscopic findings of early MGC include an elevated lesion resembling a submucosal tumor due to abundant mucin pools in the submucosa. ${ }^{18}$ In comparison to early MGC, the DMA described in the current case presented as a laterally spreading tumor at the anti-mesenteric border of the second portion of the duodenum on upper gastroendoscopy. The tumor showed no extracellular mucin or exudate. As the macroscopic appearance of the mass was not consistent with the typical endoscopic findings of mucinous adenocarcinoma, we did not predict that the lesion was a DMA, and the diagnosis was made only after the analysis of a frozen biopsy sample.

With regard to the management of sporadic duodenal adenoma, endoscopy is useful for evaluation and resection. However, in the present case, the mass involved $70 \%$ of the lumen and was too large to remove endoscopically. Because resection should be considered for lesions with $>33 \%$ circumferential involvement of the lumen, ${ }^{19}$ we chose this treatment method despite the fact that the initial biopsy result had indicated an adenoma.

Our patient had no symptoms or any relevant medical history. Most cases of SBA arise from adenomas; our patient had a DMA with a background of low-grade tubular adenoma. We detected our patient's cancer during a normal health screening and were therefore able to treat it at an early stage, unlike most cases of SBA. In conclusion, primary DMA is a particularly rare tumor and its epidemiology is not yet well defined. Herein, we have reported the first case of DMA in Korea.

Conflicts of Interest

The authors have no financial conflicts of interest.

\section{REFERENCES}

1. Siegel R, Ma J, Zou Z, Jemal A. Cancer statistics, 2014. CA Cancer J Clin 2014;64:9-29.

2. Bilimoria KY, Bentrem DJ, Wayne JD, Ko CY, Bennett CL, Talamonti MS. Small bowel cancer in the United States: changes in epidemiology, treatment, and survival over the last 20 years. Ann Surg 2009;249:63-71.

3. Yagyu T, Aihara T, Murayama M, et al. Mucinous carcinoma of the duodenum associated with hereditary nonpolyposis colorectal cancer: report of a case. Surg Today 2006;36:1129-1132.

4. Inagaki M, Obara M, Suzuki S, et al. Mucinous carcinoma of Vater's ampulla with a unique extension along the main pancreatic duct. J Hepatobiliary Pancreat Surg 2007;14:518-521.

5. Dabaja BS, Suki D, Pro B, Bonnen M, Ajani J. Adenocarcinoma of the small bowel: presentation, prognostic factors, and outcome of $217 \mathrm{pa}-$ tients. Cancer 2004;101:518-526.

6. Aparicio T, Zaanan A, Svrcek M, et al. Small bowel adenocarcinoma: epidemiology, risk factors, diagnosis and treatment. Dig Liver Dis 2014;46:97-104

7. Kummar S, Ciesielski TE, Fogarasi MC. Management of small bowel adenocarcinoma. Oncology (Williston Park) 2002;16:1364-1369.

8. Faivre J, Trama A, De Angelis R, et al. Incidence, prevalence and survival of patients with rare epithelial digestive cancers diagnosed in Europe 
in 1995-2002. Eur J Cancer 2012;48:1417-1424.

9. Chow JS, Chen CC, Ahsan H, Neugut AI. A population-based study of the incidence of malignant small bowel tumours: SEER, 1973-1990. Int J Epidemiol 1996;25:722-728.

10. Michelassi F, Testa G, Pomidor WJ, Lashner BA, Block GE. Adenocarcinoma complicating Crohn's disease. Dis Colon Rectum 1993;36:654-661.

11. Halfdanarson TR, McWilliams RR, Donohue JH, Quevedo JF. A single-institution experience with 491 cases of small bowel adenocarcinoma. Am J Surg 2010;199:797-803.

12. Abrahams NA, Halverson A, Fazio VW, Rybicki LA, Goldblum JR. Adenocarcinoma of the small bowel: a study of 37 cases with emphasis on histologic prognostic factors. Dis Colon Rectum 2002;45:1496-1502.

13. Jass JR, Sobin LH. Histologic Typing of Intestinal Tumours. 2nd ed. Berlin: Springer Verlag; 1989.

14. Yasuda K, Adachi Y, Shiraishi N, Yamaguchi K, Shiromizu A, Kitano S. Pathology and prognosis of mucinous gastric carcinoma. J Surg Oncol
2001;76:272-277.

15. Verhulst J, Ferdinande L, Demetter P, Ceelen W. Mucinous subtype as prognostic factor in colorectal cancer: a systematic review and meta-analysis. J Clin Pathol 2012;65:381-388.

16. Hirota E, Ochiai A, Oda Y, et al. Significance of histological type of gastric carcinoma as a prognostic factor. Stomach Intest 1991;26:1149-1158.

17. Adachi Y, Mori M, Kido A, Shimono R, Maehara Y, Sugimachi K. A clinicopathologic study of mucinous gastric carcinoma. Cancer 1992;69:866-871.

18. Yasuda K, Shiraishi N, Inomata M, Shiroshita H, Ishikawa K, Kitano S. Clinicopathologic characteristics of early-stage mucinous gastric carcinoma. J Clin Gastroenterol 2004;38:507-511.

19. Standards of Practice Committee, Adler DG, Qureshi W, et al. The role of endoscopy in ampullary and duodenal adenomas. Gastrointest Endosc 2006;64:849-854. 